\title{
Changes in Counseling Center Client Problems Across 13 Years
}

\author{
Sherry A. Benton, John M. Robertson, Wen-Chih Tseng, Fred B. Newton, and Stephen L. Benton \\ Kansas State University
}

\begin{abstract}
Are perceived increases in symptom severity in college counseling center clients real or imagined? Counseling center staff, retrospectively, reported that client problems are more severe now than in the past. Yet studies examining client distress levels at intake have found no significant increases. This study examined counseling center client problems across 13 years from the perspective of the treating therapist at the time of case closure. Increases were found for 14 of 19 client problem areas, whereas other areas retained stable levels, and 2 problem areas had a quadratic trend over time. These changes directly affect counseling service practices.
\end{abstract}

Across several years, counseling center staff (Robbins, May, \& Corazzini, 1985) and directors (Gallagher, Gill, \& Sysco, 2000; O'Malley, Wheeler, Murphey, O'Connell, \& Waldo, 1990) have perceived increases in levels of psychopathology and symptom severity among counseling center client populations. In these studies, therapists' and directors' responses were retrospective, reflecting their speculations about changes in client problems over the previous years. However, researchers who have attempted to examine this issue with more objective data, such as client-perceived distress scores at intake, have found no differences across 6-8 years (Cornish, Kominars, Riva, McIntosh, \& Henderson, 2000; Pledge, Lapan, Heppner, Kivlighan, \& Roehlke, 1998). How can one explain the discrepancy between therapists' and directors' post hoc subjective impressions and client-perceived distress scores? What increases in symptom severity might therapists and directors perceive that are not reflected in measures of client distress levels

SHERRY A. BENTON received her PhD in counseling psychology in 1991 from the University of Kansas. She is the assistant director for training at Counseling Services, the university's counseling center, and is a member of the graduate faculty and an assistant professor in the Department of Counseling and Educational Psychology at Kansas State University.

JoHn M. ROBERTSON received his $\mathrm{PhD}$ in counseling psychology from the University of California, Santa Barbara in 1989. He is a psychologist at Counseling Services, a member of the graduate faculty of the Department of Counseling and Educational Psychology, and an adjunct professor in the Department of Psychology at Kansas State University.

WeN-CHIH Tseng received his MEd in counseling and guidance in 1996 from National Kaohsiung Normal University, Taiwan. He is a doctoral candidate in the Department of Counseling and Educational Psychology and a graduate research assistant at Counseling Services, Kansas State University.

Fred B. Newton received his $\mathrm{PhD}$ in counseling psychology from the University of Missouri-Columbia in 1972. He is the director of Counseling Services and is a full professor in the Department of Counseling and Educational Psychology at Kansas State University.

STEPHEN L. BENTON received his PhD in educational psychology from the University of Nebraska-Lincoln in 1983. He is a full professor and chair of the Department of Counseling and Educational Psychology at Kansas State University.

CORRESPONDENCE CONCERNING THIS ARTICLE should be addressed to Sherry A. Benton, Counseling Services, Kansas State University, 232 Lafene, Manhattan, Kansas 66506-3301. E-mail: benton@ksu.edu at intake? This study attempted to lend some clarity to these questions by providing data from another perspective. We analyzed client case descriptors, completed by therapists at case closure, for each case across a 13-year period.

Several researchers have relied on retrospective survey data, asking counselors and directors to recall their impressions regarding changes in symptom severity over time. For example, Robbins et al. (1985) surveyed 200 college counseling center staffs and found that most respondents reported an increase in the emotional and behavioral problems of their clients over 3 years. Gallagher et al. (2000) completed a national survey of counseling center directors and reported that $77.1 \%$ of directors indicated that increases in the number of students with severe psychological problems were a concern for their center. O'Malley et al. (1990) also surveyed directors nationally and found similar results. These findings are quite subjective, however, and depend on directors' accurate recall of case severity over several years. It is appropriate to be cautious about the accuracy of directors' memories because they may be biased by expectations and other factors.

In studies using more objective data, researchers have found no differences in client distress levels over time. Cornish et al. (2000) examined counseling center clients' overall distress levels, across 6 years, measured at intake by having each client complete the Symptom Check List-90-R (SCL-90-R). These authors reported relatively high levels of client distress but no general changes over time. The authors did note an increase in the most seriously distressed clients in the last 2 years examined. They speculated that therapists perceived an increase in overall client severity because of an increase in the relatively small group of most distressed clients.

In another study using client-reported distress levels, Pledge et al. (1998) examined global composite scores indicating general distress using the Computerized Assessment for Psychotherapy Evaluation and Research (CASPER). Clients completed the CASPER Inventory at a computer terminal before their initial session. Although high levels of distress were prevalent across all years examined, no differences in distress level were found across 6 years (1989-1995). The authors concluded that if differences were present in past decades, they appeared to have leveled off more recently. In both of these studies (Cornish et al., 2000, and Pledge et al., 1998), the researchers relied on client self-report of symptoms at intake to derive distress scores. However, client 
distress scores at intake may not necessarily reflect client problems and client severity of symptoms as they are manifested over the course of therapy. In addition, 6-8 years is a relatively short time in which to look for long-term changes in client problems. Other researchers have found high overall scores in client distress at intake (Todd, Dean, \& McKenna, 1997; Johnson, Ellison, \& Heikkinen, 1989). Relatively high overall distress levels at intake may have differed from the therapists' perceptions of severity and client problems over the course of therapy.

In a review of the literature on client severity in college counseling centers, Sharkin (1997) identified the following five problems in these previous studies: (a) Psychopathology or severity was neither well defined nor distinguished from developmental and normal problems; (b) in prior studies, researchers reported too short of a time frame to detect trends- 3 to 8 years rather than the 10 years Sharkin suggested; (c) retrospective studies may be unreliable; (d) perhaps perceived increases in severity have been due to better training of psychotherapists, which has led to more diagnoses; and (e) clients' self-reported measures of distress may not necessarily reflect psychopathology.

The current study addressed many of the problems raised by Sharkin (1997). We used the Case Descriptor List (CDL), which measures therapists' perceptions of client developmental and relational problems along with more serious problems. Data collected from the perspective of the therapist at case closure may more accurately reflect the problems addressed in therapy and are less likely affected by expectations. In addition, this method avoids the problem of inaccurate recall that is inherent in retrospective surveys. Furthermore, data collected at termination may more directly reflect problems addressed in therapy and symptom severity than client distress levels at intake because clients can respond to life problems with varying levels of distress. For example, at intake, one client can appear very distressed by a relationship break-up, whereas another student with a significant history of serious trauma and abuse can appear only mildly anxious. Differences in distress levels would not accurately reflect differences in problem severity for these two clients.

The purpose of our study, then, was to examine trends in counseling center clients' problems from the perspective of the therapist at the time of therapy termination. We examined archival data across 13 years from a university counseling service located in a large Midwestern university.

\section{The Client Problem Study}

\section{Participants}

Participants were 13,257 student-clients who sought personal counseling at the campus counseling center on a large midwestern university campus with an average annual enrollment of about 20,000 students. All clients seen for one session or more in the counseling center were included in the study. Data collection was archival in nature and covered a span of 13 years from fiscal year 1988-1989 through 2000-2001. The beginning date was chosen because in that year two units on the campus merged: the mental health unit of the student health center and the counseling center. It was also the first year the CDL (described below) was used to collect the data analyzed in this study. The mean number of students seen annually was 1,020 , with a range of 921 to 1,078 .
The number of clients seen per year tended to be somewhat lower in years when the counseling center had an unfilled staff position or fewer interns.

The gender ratio remained fairly constant over the 13-year period. The mean percentage of female clients seen each year was $63.9 \%$, with a range of $61.1 \%$ to $67.5 \%$. During the late 1990 s, a trend toward more male clients seeking services was observed, with the percentage gradually increasing from $32.5 \%$ to $38.9 \%$ over the last 4 years.

The academic classification data showed that undergraduate students were more likely to seek services the longer they remained in school. In an average year, $16.1 \%$ of the clients were freshmen, $18.3 \%$ were sophomores, $22.7 \%$ were juniors, $26.8 \%$ were seniors, and $15.4 \%$ were graduate students. These proportions were consistent across all 13 years examined.

With regard to average age at the time of service, three of every four clients $(75.4 \%)$ were under 25 years of age, the traditional age range for university students. Ethnic group representation was similar to the ethnic proportions on campus, with students of color slightly overrepresented in the counseling service client population relative to their proportional numbers on campus. Students of color accounted for $11.8 \%$ to $14.7 \%$ of the sample across the years of the study.

In order to test whether or not there were any significant changes in demographic variables across the 13 years, we divided the sample into three groups corresponding with the counseling center fiscal years (July 1988-June 1992; July 1992-June 1996; and July 1996-June 2001) and then conducted $z$ tests for differences in population proportions. We selected three groups for two reasons. First, three time periods would allow us to detect nonlinear trends over time. Second, combining 4-5 years into a group would eliminate idiosyncratic changes. For example, we might have a large 1-year increase in clients with eating disorders as a result of media attention, high-profile cases, movies, books, and so forth. We wanted to look at more stable patterns over time rather than these 1-year increases. The three groups corresponded with the three groups used in later analyses.

These tests indicated that there were no significant differences between periods with regard to gender. However, the proportions of seniors and traditionally aged (18-25 years) students were significantly higher from 1992-1996 (.28 and .77 respectively) and from 1996-2001 (.29 and .77 respectively) than during the period 1988-1992 (.25 and .73 respectively). The proportion of White students was also significantly higher from 1988-1992 (.88) and from 1992-1996 (.88) than during the period 1996-2001 (.84). There was a corresponding shift in the proportion of students of color across time periods (.12 during the first and second time period and .16 during the third time period). Whether or not these changes in age and ethnicity are meaningful changes is open to discussion. The size of our database (more than 13,000 students) certainly increased the likelihood of finding statistically significant differences, but we were unable to hypothesize how the practical size of these shifts might have influenced any of our findings.

\section{Instrument}

The CDL was used to measure client symptoms. The CDL was developed by the counseling center staff in 1988 as a way to parsimoniously describe client problem areas addressed in coun- 
seling. The instrument was developed based on earlier instruments used by the mental health center and the counseling center before they were combined into one agency in 1988. Staff from both agencies worked together to identify pertinent categories, then the finished instrument was reviewed by the combined staff. The CDL consists of 30 questions answered by the clinician on the computer at the time each case is closed. Nineteen of the items refer specifically to client problem areas; the other items address the final disposition of the case (referral to another agency, withdrawal from school, and so forth). All items are dichotomous, with the therapist simply checking yes or no for each item. Because many cases involve multiple problems, the therapist checks yes for all items that apply to that case. In effect, the list provides a frequency count of client problems addressed during therapy. The items are phrased in general terms, not in the language of the Diagnostic and Statistical Manual of Mental Disorders (4th ed.; DSM-IV; American Psychiatric Association, 1994). Examples of these broad categories in the CDL include "relationship issues," "depression," "physical problems," and "personality disorders." The CDL was used in each of the 13 years, and no changes were made in it over those years. Interns and new staff received training on the instrument during orientation at the start of each academic year.

To test the reliability of the CDL, 11 staff members were given three actual case summaries from the agency's files. These three cases were selected at random. Names of both client and therapist were blocked out. Each case summary presented information about the presenting problem, the context (academic history, mental status, and so forth), problem history, family history, any test results, and a treatment summary. The 11 therapists were asked to read each case summary and then complete the CDL as though the case were their own. Raters checked either yes or no to indicate the presence or absence of each characteristic. Then, for each of the 30 characteristics, we divided the number of agreements by the number of raters to calculate $P_{\mathrm{A}}$, the percentage of agreement (Ebel \& Frisbie, 1991). The grand mean for $P_{\mathrm{A}}$ across the three cases was .91 , indicating that $91 \%$ of the decisions about the characteristics of the three cases were consistent across the 11 raters.

\section{Clinicians}

The clinicians in this study were 12 full-time (or nearly fulltime) staff members at the counseling center. Eleven were $\mathrm{PhD}$ psychologists, and one was a master's-level counselor. Very few staff changes occurred across the 13 years. To calculate a staff stability index, we first computed the average percentage of staff members who remained on staff from one year to the next (based on a strategy developed by van Vugt, 2001). The average percentage of carryover each year was $94 \%$. Because we were especially interested in whether or not there was a significant change in staff composition between the two time periods of our study (19881994 vs. 1995-2001), we then used the chi-square test to estimate stability between the two groups. Using Yate's correction for continuity, the results indicated no significant differences in staff composition between the two time periods, $\chi^{2}(1, N=12)=0.167$, $p<.05$.

In addition to the 12 clinicians, the counseling center employed three or four predoctoral psychology interns each year. Given that this cohort changed each year, it was assumed that their distribution between the earlier and later groups would be random and would therefore be unlikely to contribute significantly to any differences between the two time periods.

Other factors that contributed to the stability of the clinical staff included no changes in the university administrators responsible for the counseling center, no changes in the positions of the counseling center director or clinical director, no change in location, and no large programmatic changes.

\section{Procedure}

Data were analyzed to determine if there were changes in symptom severity across three time periods. Because the fiscal year ended each year in June, the first group included cases from July 1, 1988 through June 30, 1992; the second group from July 1, 1992 through June 30, 1996; and the third group from July 1, 1996 through June 30,2001 . We conducted a series of $3 \times 2$ chi-square tests, followed by post hoc $2 \times 2$ tests of association for any significant initial $3 \times 2$ analyses. Given the very large sample size, we selected a relatively conservative alpha level by dividing .05 by 19 , the number of client problem areas analyzed from the CDL. Type I error rate was therefore set at .003 for all analyses.

\section{Results and Discussion}

We conducted a series of $3 \times 2$ chi-square tests of association to determine if there were significant changes in client problems across three periods: 1988 to 1992, 1992 to 1996, and 1996 to 2001. Our subjective impression was that the numbers of students seen for a specific problem area could sometimes spike in a single year because of external factors, such as media attention to a problem or a high-profile case making the news. Three groups would likely decrease the likelihood of detecting changes attributable to these external factors but would detect the pattern of more enduring trends. Table 1 presents the results of those tests and percentages of individuals experiencing each problem area across the three time periods. Significant changes in percentages were observed in 14 of the 19 problem areas. We followed up these significant $3 \times 2$ chi-square tests with $2 \times 2$ analyses to make pairwise comparisons across each of the three time periods. To control for the possibility of Type I error inflation that accompanies multiple comparisons, we used the Bonferroni technique to adjust the alpha level by dividing .003 by 3 , the number of pairwise comparisons. The per-comparison alpha was therefore set at .001

As indicated in Table 1 and in Figure 1, relatively linear trends were observed in six of the problem areas: developmental, situational, depression, academic skills, grief, and medication use. The percentage of students experiencing these problems increased steadily across the three time periods. In seven of the other problem areas (i.e., relationships, stress/anxiety, family issues, physical problems, personality disorders, suicidal thoughts, and sexual assault), the percentages of students experiencing difficulties was greater during the latter two time periods than in the first time period (see Figures 2 and 3). Overall, these results indicate that in 14 of the 19 problem areas, clinicians reported increases in the percentages of individuals having difficulties. Of particular interest is that, up until 1994, relationship problems were the most frequently reported client problem (see Figure 3). During 1994, however, stress/anxiety problems were reported more frequently 
Table 1

Percentages of Clients Experiencing Each Problem Area Across Three Time Periods

\begin{tabular}{lcccrr}
\hline \multicolumn{1}{c}{ Client problem } & $\begin{array}{c}1 \\
(\%)\end{array}$ & $\begin{array}{c}2 \\
(\%)\end{array}$ & $\begin{array}{c}3 \\
(\%)\end{array}$ & $\begin{array}{c}\text { Pearson } \\
\chi^{2}\end{array}$ & $\begin{array}{c}\text { Post hoc } \\
\text { comparison }\end{array}$ \\
\hline Relationship & 46.54 & 57.25 & 56.16 & 117.22 & $2,3>1^{*}$ \\
Stress/anxiety & 36.26 & 63.42 & 62.87 & 825.51 & $2,3>1^{*}$ \\
Family issues & 32.87 & 42.85 & 44.82 & 148.03 & $3,2>1^{*}$ \\
Situational & 21.91 & 43.19 & 58.24 & $1,234.02$ & $3>2>1^{*}$ \\
Educational/vocational & 21.76 & 17.12 & 21.74 & 37.02 & $1,3>2^{*}$ \\
Depression & 21.10 & 34.49 & 40.67 & 404.64 & $3>2>1^{*}$ \\
Developmental & 18.98 & 28.99 & 41.41 & 547.24 & $3>2>1^{*}$ \\
Abuse & 11.70 & 15.40 & 12.31 & 28.69 & $2>1,3^{*}$ \\
Medication used & 8.97 & 12.04 & 22.22 & 354.22 & $3>2>1^{*}$ \\
Academic skills & 8.50 & 24.66 & 34.46 & 860.41 & $3>2>1^{*}$ \\
Physical problems & 6.58 & 11.79 & 13.52 & 118.90 & $2,3>1^{*}$ \\
Substance abuse & 6.38 & 6.39 & 6.64 & 0.33 & \\
Eating disorders & 5.26 & 6.00 & 5.59 & 2.07 & $2,3>1^{*}$ \\
Personality disorders & 2.61 & 6.12 & 7.23 & 99.12 & $2,3>1^{*}$ \\
Suicidal & 4.80 & 9.01 & 8.98 & 70.01 & $3>2>1^{*}$ \\
Grief & 4.41 & 8.01 & 10.23 & 108.38 & \\
Chronic mental disorder & 2.90 & 2.44 & 3.49 & 8.70 & $2,3>1^{*}$ \\
Legal & 2.12 & 3.06 & 3.00 & 8.75 & \\
Sexual assault & 0.17 & 3.66 & 3.45 & 132.54 & \\
\hline
\end{tabular}

Note. 1 refers to the years $1988-1992(N=4,104) ; 2$ refers to $1992-1996(N=4,019) ; 3$ refers to $1996-2001$ $(N=5,134)$.

$* p<.001$.

and have remained more frequent than relationship problems in all subsequent years.

In contrast to the problem areas evidencing linear increases, two of the problem areas revealed significant quadratic trends (see Figure 4). Educational/vocational problems were more prevalent in the first time period than in the second and then increased again in the third. In contrast, problems of abuse increased from the first to the second time period and then dropped off in the third. No significant changes were observed for substance abuse, eating disorders, legal problems, or chronic mental disorders.

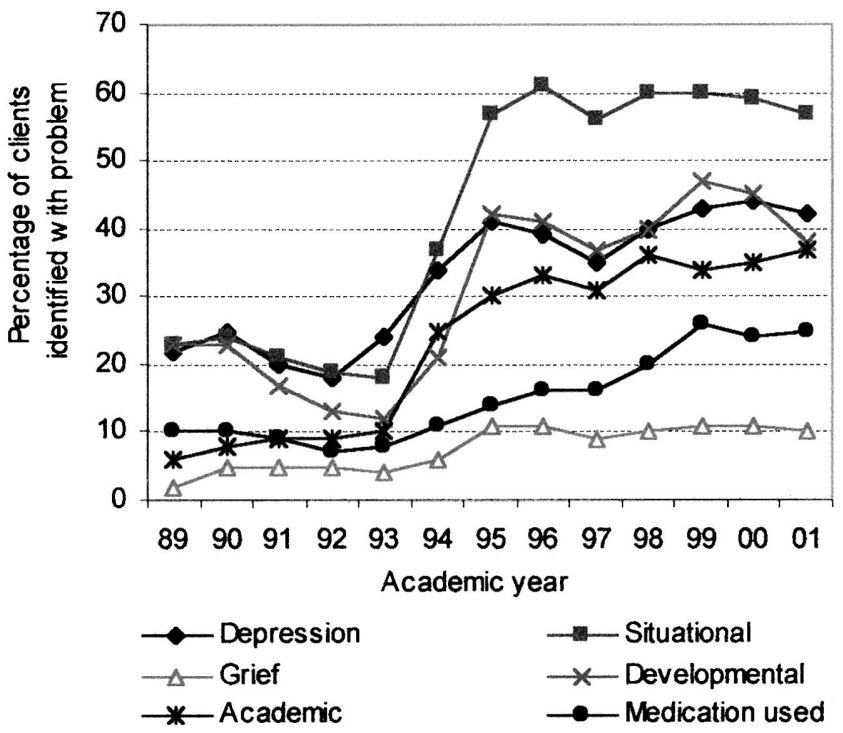

Figure 1. Client problems with linear increases over time.
Overall, our results indicated that students who were seen in counseling services in more recent time periods frequently have more complex problems that include both the normal college student problems, such as difficulties in relationships and developmental issues, as well as the more severe problems, such as anxiety, depression, suicidal ideation, sexual assault, and personality disorders. Some of these increases were dramatic: The number of students seen each year with depression doubled over the time period, while the number of suicidal students tripled and the

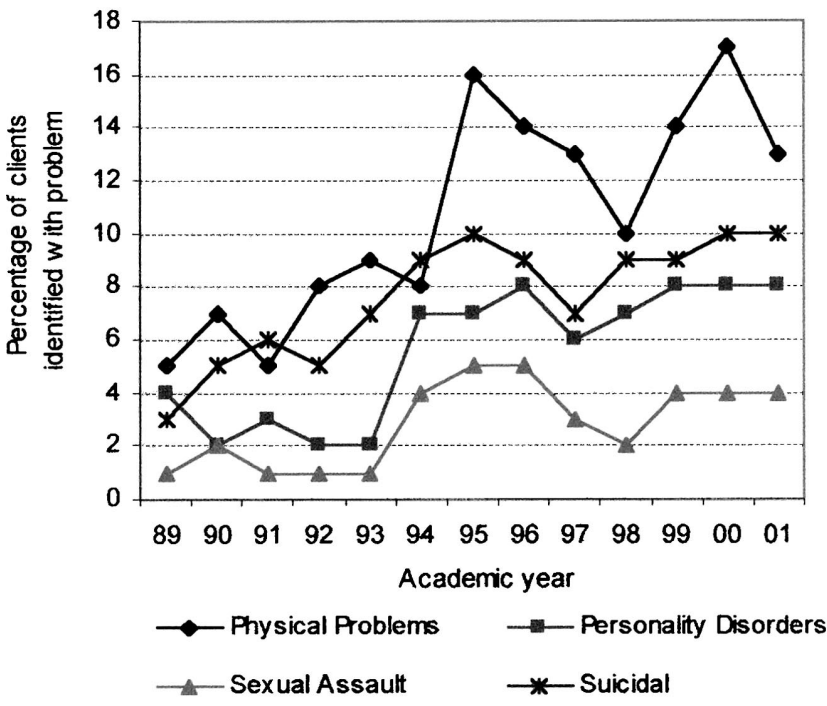

Figure 2. Client problems with a low base rate, where Time Period $1<$ Time Periods 2 and 3. Time Period $1=1988-1992$; Time Period $2=$ 1992-1996; Time Period $3=1996-2001$. 


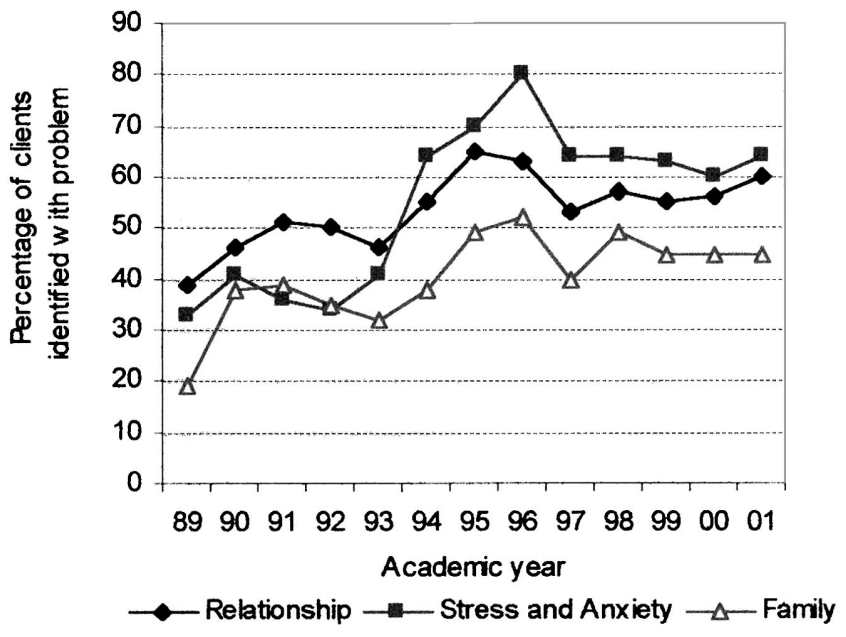

Figure 3. Client problems with a high base rate, where Time Period $1<$ Time Periods $<2$ and 3. Time Period $1=1988-1992$; Time Period $2=$ 1992-1996; Time Period 3 = 1996-2001.

number of students seen after a sexual assault quadrupled. The pattern of change was also of interest, as some problem areas showed steep increases from the first time period to the second and then appeared to stabilize from Time Period 2 to Time Period 3. Relationship problems, stress/anxiety, family issues, physical problems, personality disorders, suicidal, and sexual assault all followed this pattern. In addition, we found significant increases in several problem areas more commonly expected in college counseling centers, including developmental problems, relationship problems, difficulties with academic skills, and situational problems.

The pattern of these changes in client problems over time has been complex. Several problems likely viewed by most counseling center staff as severe-substance abuse, eating disorders, legal problems, and chronic mental illness-showed no significant change over the 13 years of the study. The base rates for both legal problems and chronic mental illness were quite low; consequently, we were less likely to find significant increases, although the numbers of students with legal problems and with chronic mental illness have increased somewhat. Proportions of students with substance abuse and eating disorders remained remarkably stable over the three time periods. This may be because therapists tended to identify students with substance abuse and eating disorders only at levels diagnosable by the $D S M-I V-T R$ (text rev.; American Psychiatric Association, 2000). Some students who have had an alcohol or substance-related problem might be identified as having a situational problem if the problem was not indicative of a pattern of abuse or was not sustained over time and, as a result, did not warrant a diagnosis of substance abuse or dependence. Consequently, it appears that these problems have remained stable in our client population across all 13 years, although substance-related situational problems may have varied from year to year. It is interesting to note that situational problems increased quite dramatically over the time periods. One weakness in the CDL is the lack of specificity with regard to the nature or types of situational problems.

Significant changes were also observed in abuse (physical, sexual, and emotional), which increased between the early and middle time periods and then declined during the third time period. Educational/vocational choice also declined during the middle time period and increased in the more recent time period.

Many variables may have led to changes in client problems, including factors idiosyncratic to our campus, changes in the larger community, media and popular press influences, and changes in psychology and mental health practice overall. We suggest the following contextual or environmental factors may also have had an effect on our results.

\section{Contextual and Environmental Factors}

The period studied coincided with the rise of managed care in the larger mental health world. Although the counseling center staff rarely had to deal with insurance companies, managed care affected the counseling service by leading to a change in policy from little attention to the number of sessions each client received during the early years of the study, to focusing on brief therapy, particularly from 1993 to 1995 and continuing through the latter years. We remained cautious about assigning diagnoses to clients but were more aware of using time efficiently and working within 4-10 sessions. Consequently, although the numbers of students with more serious problems - such as stress and anxiety, sexual assault, depression, suicidal ideation, and personality disordersincreased, the focus of the counseling service was on limiting student counseling sessions to 10 or fewer. Over the years of the study, the average number of sessions per client decreased over time: From 1989-1992, the mean number of sessions was 6.87; from 1992-1996, the mean was 6.17; and from 1996-2001, the mean was 5.98. In addition, we referred more clients out to other community resources during the later time periods. Another indicant of this trend was the steep increase from 1993 to 1995 in clients identified as having "situational problems". During this period the counseling center began to focus on moving to more short-term work; consequently, therapists appeared to have focused more on the situational or immediate aspects of students' problems, in the interest of working within a brief model. For example, a student could come in to the counseling center with a history of physical abuse and symptoms of posttraumatic stress,

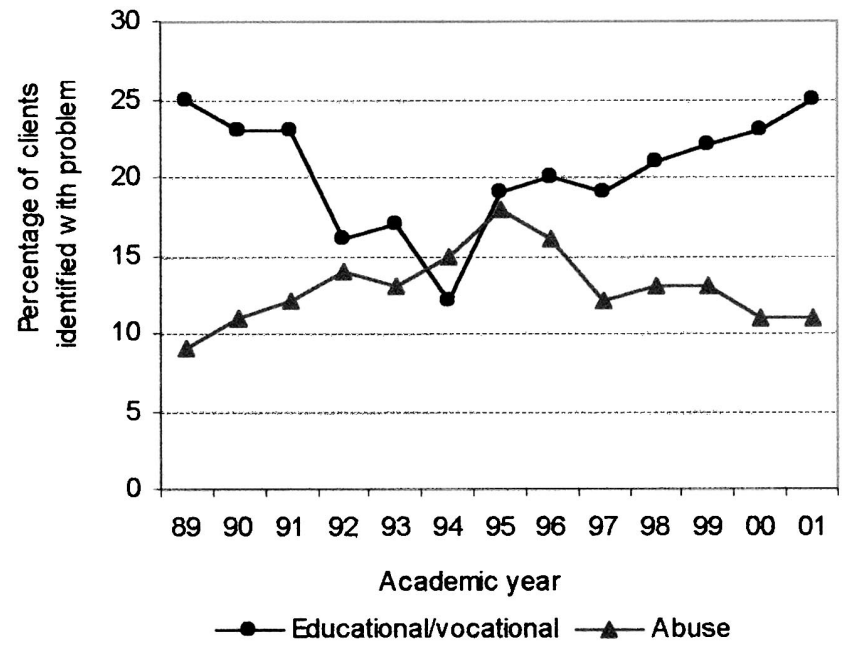

Figure 4. Client problems with a quadratic pattern over time. 
but the immediate precipitator for seeking counseling was anxiety and panic he experienced while in class. In the earlier time period, this student would likely have been identified with several case descriptors, including "abuse," "stress and anxiety," and "family issues." The problem of anxiety and panic would have been viewed within the context of the abuse and situational stress and would not have been seen as the primary focus of therapy. During the later time periods, the therapist would more likely also identify the anxiety and panic in class as "situational problems" (along with the other descriptors) and would focus more on these immediate issues, because these might be more amenable to very brief interventions than would the long-term effects of abuse.

Another environmental factor may have affected the quadratic pattern in educational and vocational choice. Through the early years of the study, the counseling service was the only university department to offer career and vocational counseling, and 22-25\% of clients who were seen reported problems with career and vocational choice. In 1993, the university opened the Academic and Career Information Center (ACIC) as a method of providing career guidance to larger numbers of students. In the first few years after the ACIC opened, the counseling center saw fewer clients with career and vocational concerns. One year only $12 \%$ of clients reported this as a problem. During the most recent time period, the numbers of clients seen for career and vocational decision making returned to the levels before ACIC was opened-25\% in 2001. The ACIC continues to work with large numbers of students as well, so this increase seems to reflect real increases in students struggling with career and vocational indecision.

The quadratic pattern in abuse is more difficult to explain. One hypothesis is that the popularity of several books, made-for-TV movies, and sustained attention to childhood physical and sexual abuse in the media may have led to increases in clients presenting with these concerns from 1994-1997. As media attention shifted to "false memories" and skepticism about reports of abuse, the number of students presenting with abuse histories may have declined. An alternative explanation might be that concerns about false memories and lawsuits against therapists may have led us to be less likely to identify a client as a victim of abuse.

Frequency of medication use represented an interesting irony. It is important to note that counselors check "medication used" only when this was part of the clients' treatment in the counseling center. Some of these clients began using medication prior to coming to the university, but the category was checked only if this medication was considered part of their continuing treatment. From 1989-1995, the counseling center employed a psychiatrist 40 hours per week. After 1995, we contracted psychiatric care 8 hours per week. Yet across the 13 years, the percentage of clients prescribed medication increased from $10 \%$ of clients to $25 \%$ of clients. This may, in part, be related to primary care physicians becoming more comfortable prescribing psychotropic medications and to our increased comfort with referring clients to primary care physicians for medication consultations as an adjunct to psychotherapy. Our subjective impression has been that the number of students coming in already using medications, particularly antidepressants, has increased, but we do not have data to support this impression.

As a research team we considered alternative explanations for the trends observed. We concluded that the most parsimonious explanation was that real shifts occurred in client problems ad- dressed in therapy, along with some change in our attention to brief therapy and the press to resolve presenting concerns in 10 sessions or fewer. Changes in training or qualifications of staff cannot account for the observed differences. All of our psychologists were familiar with $D S M-I V$ diagnosis throughout the 13-year period. Training in diagnosis for our predoctoral psychology interns increased in 2000 and 2001; however, this does not account for the differences observed.

\section{Implications for Our Counseling Center}

We have made several adaptations within our counseling center as a result of the observed changes in client problems. First, the percentage of time we spend doing individual psychotherapy has actually decreased relative to the percentage of time we spend on report writing and consultation with referral sources, doctors, hospitals, other student services, academic departments, and families. For example, across the years of this study the number of students who were seen each year for suicidal ideation or intent tripled. Providing effective support to suicidal students, in order to ensure their safety and their ability to function as a student, often required psychologists to work with the student's family, residence hall staff, academic department, psychiatrists and health center staff, and other student services. Providing support and ensuring the safety of a suicidal student was often time intensive. Despite increases in clients with more complex and severe problems, there have been no corresponding adjustments in the number of clinical staff over this time period. Second, the emphasis on diagnostic and assessment skills, comprehensive and complete record keeping, and case management have all increased. Third, when new positions have opened up, we have looked for psychologists with experience working with more severe populations; this has also been true in selecting interns.

Training priorities have also changed. Crisis work is now much more of a priority than it was a decade ago. Interns now routinely learn to give mental status exams and to do rapid assessment. We begin to introduce interns to referral sources, such as the psychiatric section of the local hospital, early in orientation. Emphasis on training with the $D S M-I V-T R$ has increased as well. Supervisors have found a greater need to be available outside of scheduled supervision hours to consult with interns on more complex and severe cases

More complex and severe client cases have created dilemmas for our counseling center staff. Although we have not adopted a session limit, we have worked from a brief model, which, at times, can feel incongruent with client needs. On the other hand, working with clients long term is impractical. As the number of sessions per client increases, students can quickly reach the point where they are receiving, dollar for dollar, more in psychological services than they paid in tuition and fees. This is not financially viable, even if clients are charged a considerable per-session fee. With session limits and managed care pressure outside of the counseling center, referral to other resources may not provide better options.

\section{Implications for Other Counseling Centers}

Three implications of the current findings are apparent: First, these findings provide empirical support for the idea that counseling centers may, in fact, be seeing clients with more complex and 
severe problems in some problem areas than they did a decade ago. If these observed patterns of change prove to be consistent with those at other counseling centers, then it is evident that therapists in counseling centers are seeing students with more critical needs than a decade ago. Students who are seen in counseling centers today frequently have more complex problems that include both the normal college student problems, such as difficulties in relationships and developmental issues, along with more severe problems, such as anxiety, depression, suicidal ideation, and personality disorders. Students with more complex and severe problems often require more resources to support them. However, with attention to brief therapy and session limits, coupled with the diminishing resources in the community, students find fewer options for counseling and mental health care available. This leaves the role of providing care primarily in the hands of counseling center staff.

Second, examining the trends in client problems can be helpful in counseling center program planning. Knowing which problems appear to be relatively stable and which seem to be increasing or decreasing provides valuable information, allowing staff to shape services to best meet the needs of students. Therapists and administrators may also need to reconsider session limits, referral sources, and staffing and funding for counseling services in the light of these more complex and severe client problems.

Third, changes in counseling center client problem severity and complexity affect how psychologists are prepared to work in counseling center settings. For example, understanding diagnosis is far more important today than it was in 1989. Conceptualizing clients with multiple problems, within a cultural context, with attention to idiosyncratic and social and environmental factors is important. As the severity and complexity of clients' problems increase, the need to work with a team of professionals becomes more important. Psychologists need to be prepared to work with physicians, social workers, other campus departments, and other health care professionals. Training in consultation is more important now than it might have been a decade ago. Although we did not collect data on crisis and trauma situations, the observed increases in the numbers of suicidal students suggest crisis intervention and trauma debriefing would also be an important area of training.

\section{References}

American Psychiatric Association. (1994). Diagnostic and statistical manual of mental disorders (4th ed.). Washington, DC: Author.

American Psychiatric Association. (2000). Diagnostic and statistical manual of mental disorders (4th ed., text rev.) Washington, DC: Author.

Cornish, J. A., Kominars, K. D., Riva, M. T., McIntosh, S., \& Henderson, M. C. (2000). Perceived distress in university counseling center clients across a six-year period. Journal of College Student Development, 41, $104-109$.

Ebel, R. L., \& Frisbie, D. A. (1991). Essentials of educational measurement (5th ed.). Englewood Cliffs, NJ: Prentice Hall.

Gallagher, R. P., Gill, A. M., \& Sysco, H. M., (2000). National survey of counseling center directors 2000. Alexandria, VA: International Association of Counseling Service.

Johnson, R. W., Ellison, R. A., \& Heikkinen, C. A. (1989). Psychological symptoms of counseling center clients. Journal of Counseling Psychology, 36, 110-114.

O'Malley, K., Wheeler, I., Murphey, J., O'Connell, J., \& Waldo, M. (1990). Changes in levels of psychopathology being treated at college and university counseling centers. Journal of College Student Development, 31, 464-465.

Pledge, D. S., Lapan, R. T., Heppner, P. P., Kivlighan, D., \& Roehlke, H. J. (1998). Stability and severity of presenting problems at a university counseling center: A 6-year analysis. Professional Psychology: Research and Practice, 29, 386-389.

Robbins, S. B., May, T. M., \& Corrazini, J. G. (1985). Perceptions of client needs and counseling center staff roles and functions. Journal of Counseling Psychology, 32, 641-644.

Sharkin, B. S. (1997). Increasing severity of presenting problems in college counseling centers: A closer look. Journal of Counseling and Development, 75, 275-281.

Todd, D. M., Deane, F. P., \& McKenna, P. A. (1997). Appropriateness of SCL-90-R adolescent and adult norms for outpatient and non-patient college students. Journal of Counseling Psychology, 44, 294-301.

van Vugt, M. (2001, July). Team stability and performance in premier league football [Study reported in M. van Vugt, Should I stay or should I go? Membership stability in social dilemmas]. Paper presented at the London School of Economics, London, England.

Received February 18, 2002

Revision received September 3, 2002

Accepted September 18, 2002 